\title{
RELACIÓN ENTRE FACTORES DE RIESGO DEL CONSUMO DE DROGAS Y PRONÓSTICO ACADÉMICO EN ESTUDIANTES DE NUEVO INGRESO EN LA CARRERA DE ENFERMERÍA EN DOS UNIVERSIDADES LATINOAMERICANAS
}

\section{CONNECTION BETWEEN RISK FACTORS ARISING FROM LICIT AND ILLICIT DRUG CONSUMPTION AND ACADEMIC PROGNOSIS IN FIRST YEAR NURSING}

STUDENTS FROM TWO LATINOAMERICAN UNIVERSITIES

"Navarro de Sáez, M., ${ }^{* *}$ Cometto, MC., ${ }^{* * *}$ Aespig, H., ${ }^{* * * *}$ Cromaguera, F.,

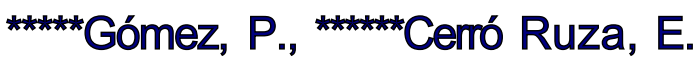

*Directora del Centro de Estudios sobre el Problema de las Drogas. Universidad de Carabobo. **Profesora Adjunta de Enfermería en Alto Riesgo. Escuela de Enfermería. Facultad de Ciencias Médicas. Universidad Nacional de Córdoba. ***Profesor Titular. Escuela de Salud Pública. Facultad de Ciencias de la Salud. Universidad de Carabobo. ****Psicóloga. Centro de Estudios sobre el Problema de las Drogas. Universidad de Carabobo. ******Profesora Adjunta de Investigación en Enfermería. Escuela de Enfermería. Facultad de Ciencias Médicas. Universidad de Córdoba. $* * * * *$ Socióloga. Centro de Estudios sobre el Problema de las Drogas. Universidad de Carabobo.

Palabras clave: Factores de Riesgo, Pronóstico Académico, Estudiantes Universitarios. Keywords: Risk Factors, Academic Prognosis, and University Students.

\section{RESUMEN}

El objetivo del estudio fue comparar los factores de riesgo del consumo de drogas y el pronóstico académico en estudiantes de nuevo ingreso de la carrera de enfermería en dos universidades latinoamericanas. El diseño fue no experimental, de tipo transeccional, descriptivo-correlacional, se aplicaron dos instrumentos: el Test de Pronóstico académico (APT) de Bennett y la prueba DUSI (Drug Use Screening Inventory) de la OEA/CICAD. Los hallazgos evidenciaron que el pronóstico académico fue diferente en ambos países, y los factores de riesgo mas relevantes para el consumo de drogas fueron la recreación y el uso del tiempo libre para estudiantes de ambas universidades. Se encontró que la presencia de un razonamiento abstracto es un factor protector del consumo de drogas, se concluye en la necesidad de crear programas preventivos, basados en el enfoque de habilidades psicosociales, que desarrollen en los estudiantes la capacidad de pensar y tomar decisiones como una habilidad para la vida que les permitiría resistir las presiones de grupos propias de la edad. 


\section{ABSTRACT}

The objective of this study was to compare risk factors associated with licit and illicit drugs use and academic prognosis of first year nursing students from two Latin American universities. The research design was non-experimental, transectional, and correlational-descriptive. Two questionnaires were used: Academic Prognosis Test (APT) by Bennett and Drug Use Screening Inventory (DUSI) test by OEA/CICAD. The outcome indicates that the academic prognosis was different in both countries, and the most relevant risk factors associated with licit and illicit drugs abuse were recreation and free time use in students from both universities. It was found that abstract reasoning ability is a drug consumption protective factor. It is concluded that it is relevant to create preventive programs, based on psychosocial skills that make students develop thinking abilities and decision making as a life skill that helps them to withstand pressures from peer groups.

\section{INTRODUCCIÓN}

El fenómeno del consumo de drogas constituye un hecho complejo que abarca aspectos tan diversos y variados como los personales, familiares y sociales, con gran incidencia en la salud, la educación y la seguridad pública. Dicho fenómeno es un problema que se ha extendido a gran parte de la población de los países latinoamericanos, afectando especialmente a los jóvenes, mostrando índices crecientes en los niveles de consumo de tal población. En tal sentido, se ha observado que un alto porcentaje de adolescentes y jóvenes en América Latina han reportado niveles de consumo de alcohol superiores, en la mayoría de los casos, al $85 \%$ en las primeras etapas de la juventud (1).

Estudios realizados a jóvenes en etapas de educación superior han arrojado prevalencias de consumo considerables que inclinan a reflexionar sobre la dinámica del fenómeno del consumo de drogas en las universidades. A modo de ejemplo se pueden citar para el caso de Argentina, niveles de prevalencia de consumo actual en fumadores de $31,75 \%$ en estudiantes de Medicina en la Universidad Nacional del Nordeste (2); mientras que estudiantes de Ciencias de la Salud de Lima mostraron tasas de 38,7\% de consumo de tabaco, así como de $94,3 \%$ para consumo de alcohol; $16,7 \%$ para el consumo de marihuana; $7,5 \%$ para pasta base y cocaína de 7,6\% (3). En México, adolescentes de educación media superior presentaron resultados superiores a $5 \%$ en prevalencia de vida respecto al consumo de tabaco y alcohol, y $7 \%$ respecto a la marihuana (4). En el caso de Venezuela, estudiantes de la escuela de Arquitectura mostraron una prevalencia de consumo de alcohol y drogas lícitas de $71,5 \%$ así como de $2,5 \%$ para el consumo de drogas ilícitas (5).

El esbozo presentado en cuanto al consumo en jóvenes invita a reflexionar respecto a los efectos adversos asociados al consumo de estas sustancias, los cuales abarcan aspectos de la salud física, psicológica y social de las personas consumidoras; es así como el consumo de drogas desde temprana edad va afectando el sistema nervioso central, con las conocidas repercusiones negativas en los neurotransmisores y las funciones psicológicas básicas, controladas por el cerebro. Estos procesos psicológicos básicos, como la atención, la memoria y el pensamiento, son indispensables para obtener un buen desempeño académico, tal como se ha demostrado en la literatura, en la que el rendimiento académico obedecen factores intelectuales, así como otros factores como la aptitud para el estudio y la personalidad (6).

Según estudios entre el año 2004 y 2006, en varios países de América Latina, dirigidos a jóvenes de educación secundaria, se ha considerado al rendimiento académico como un factor clave, tanto de riesgo como de protección, en materia de consumo de drogas, 
teniendo como resultado que "aquellos estudiantes que presentan bajas calificaciones y/o han repetido algún año escolar, presentan tasas de consumo de drogas más altas que quienes muestran mejor rendimiento estudiantil" (7). En una investigación realizada a estudiantes colombianos, se evaluaron los factores que estuvieron incidiendo en el desempeño académico de los estudiantes de primer semestre de medicina, y los resultados obtenidos en la prueba de aptitudes diferenciales y generales (BADyGs) del aprendizaje fueron bajos. El análisis mostró que el no leer como pasatiempo, la presencia de violencia intrafamiliar, el haber fumado marihuana, el provenir de un colegio mixto, el no haber realizado estudios profesionales, de quién se depende económicamente, las notas de biología, bioquímica y del promedio trimestral fueron los factores que se asocian con fracaso académico o pérdida de cupo (8).

No deja de ser preocupante situaciones como la reseñada al relacionarla con los procesos de aprendizaje en educación superior, especialmente cuando en América Latina fenómenos asociados al rendimiento académico, tales como la deserción y repitencia muestran tasas sumamente elevadas. Para el caso de la educación superior en Venezuela, se han observado niveles considerables de repitencia y deserción académica, especialmente en aquellas carreras asociadas con mayores niveles de dificultad y exigencia hacia sus estudiantes. En este sentido, se manejan cifras muy alarmantes, por ejemplo, el cincuenta por ciento de los estudiantes que llega a la universidad fracasa, además que el fenómeno de la repitencia se presenta en los cuatro primeros semestres de las carreras universitarias" (9)

La población de nuevo ingreso a las universidades constituye una oportunidad para explorar la problemática de las drogas; es por ello que se plantea la realización de una investigación para analizar la relación entre los factores de riesgo para el consumo de drogas y el pronóstico académico en estudiantes de nuevo ingreso a la carrera de enfermería en dos universidad latinoamericanas.

\section{MATERIALES Y MÉTODOS}

La investigación fue de tipo descriptivo-correlacional (10) y el diseño observacional, transversal (11), debido a que se indaga sobre la incidencia de las modalidades o niveles de una o más variables en una población, y se describe la relación entre dos o más categorías, conceptos o variables en un momento determinado (10). La población estuvo constituida por 152 estudiantes cursantes de la Universidad de Carabobo, Venezuela, y 182 estudiantes de la Universidad Nacional de Córdoba, Argentina, cursantes todos del primer año de la carrera enfermería. La muestra se conformó por 23 estudiantes de cada universidad. La selección de los participantes en la investigación fue no probabilística, de voluntarios, previo consentimiento informado. Para ello se aplicaron dos instrumentos de recolección de datos: en primera instancia, el Test de Pronóstico Académico (APT) de Bennett, una batería construida como un test de elementos sencillos que se adapta a diferentes niveles escolares, se utilizó la versión española que está conformada por 3 pruebas: razonamiento abstracto, razonamiento verbal y razonamiento numérico (12). Estas pruebas permiten apreciar las aptitudes intelectuales que más se correlacionan con el éxito académico. La prueba verbal esta constituida por 60 elementos a base de analogías lo que constituye una forma de razonamiento e implica e tanto el manejo de información como la agilidad para manejar conceptos y palabras. La prueba numérica se compone de 60 elementos relativamente heterogéneos que representan una muestra de varias aptitudes numéricas, requieren la compresión de conceptos matemáticos y la habilidad para el cálculo aritmético y la capacidad de razonamiento con esta clase de material cuantitativo. La prueba de razonamiento abstracto es descrita como un test de 
clasificación de figuras, y requiere que el sujeto descubra el principio en virtud del cual 3 figuras poseen unas características en común y que reconozca cuál de las otras figuras propuestas participa de las mismas características (13)

EI segundo instrumento utilizado fue la PRUEBA DUSI (Drug Use Screening Inventory), elaborado por el doctor Ralph Tarter (14), y recomendado por la OEA/CICAD para la medición de factores de riesgo del consumo de alcohol y otras drogas en adolescentes, consta de 82 ítemes relacionados con los factores de riesgo del consumo de drogas, distribuidas en 10 áreas o dominios: conducta, estado emocional, competencia social, familia, académica, grupo de pares, socio-culturales, recreación, uso del tiempo libre y consumo problemático de drogas. Los resultados del instrumento arrojan un índice de severidad, el cual da cuenta del porcentaje de respuestas positivas en la muestra evaluada; en tal sentido, se obtiene un índice de severidad por área para los ítemes de cada dominio, y un índice de severidad global para todas las áreas del instrumento. El valor crítico estimado tanto para los índices de severidad global como índices de severidad por área, reflejan el porcentaje de la muestra evaluada cuyo índice de severidad se encuentra por encima del $30 \%$, es decir, se encuentra en situación de riesgo del consumo de alcohol y otras drogas al tener más del $30 \%$ de respuestas positivas.

Para conocer el tipo de relación estadística existente se analizaron los datos a través del coeficiente de correlación de Pearson. El análisis de los datos obtenidos se realizó a través del programa estadístico SPSS, versión 12.

\section{RESULTADOS}

En primera instancia, se analizó el pronóstico académico como un factor de riesgo de consumo de drogas, en el análisis se obtuvieron diferencias entre ambos países (Gráfico $\mathrm{n}^{\circ}$ 1). En la subprueba de razonamiento abstracto los y las estudiantes venezolanos obtuvieron una media aritmética de 32,87 y desviación estándar ( \pm ) de 11,05; mientras que los estudiantes argentinos obtuvieron media aritmética de $14,32 \pm 2,65$. En la prueba de razonamiento verbal, los estudiantes venezolanos obtuvieron una media aritmética de $23,09 \pm 9,23$; mientras que los y las estudiantes argentinos obtuvieron una media aritmética de $28,03 \pm 7,0$. En la subprueba razonamiento numérico, los estudiantes venezolanos obtuvieron una media aritmética $17,68 \pm 11,18$; los estudiantes argentinos presentan una media aritmética de $26,70 \pm 7,95$. No se observaron diferencias estadísticamente significativas en las puntuaciones obtenidas en las subpruebas, y en cuanto a la puntuación total del pronóstico académico se encontró diferencias en la puntuación obtenida por las y los estudiantes en las subpruebas de Pronóstico Académico, en las cuales los y las estudiantes venezolanos obtuvieron una media de puntuación total correspondiente a 74,9 , mientras las y los estudiantes argentinos obtuvieron una media de puntuación total de 69,2; sin embargo, esta diferencia no fue estadísticamente significativa. 


\section{Gráfico $\mathrm{N}^{\circ}$ 1. Pronóstico académico según puntuaciones en las sub-pruebas ATP en}

estudiantes venezolanos(as) y argentinos(as)

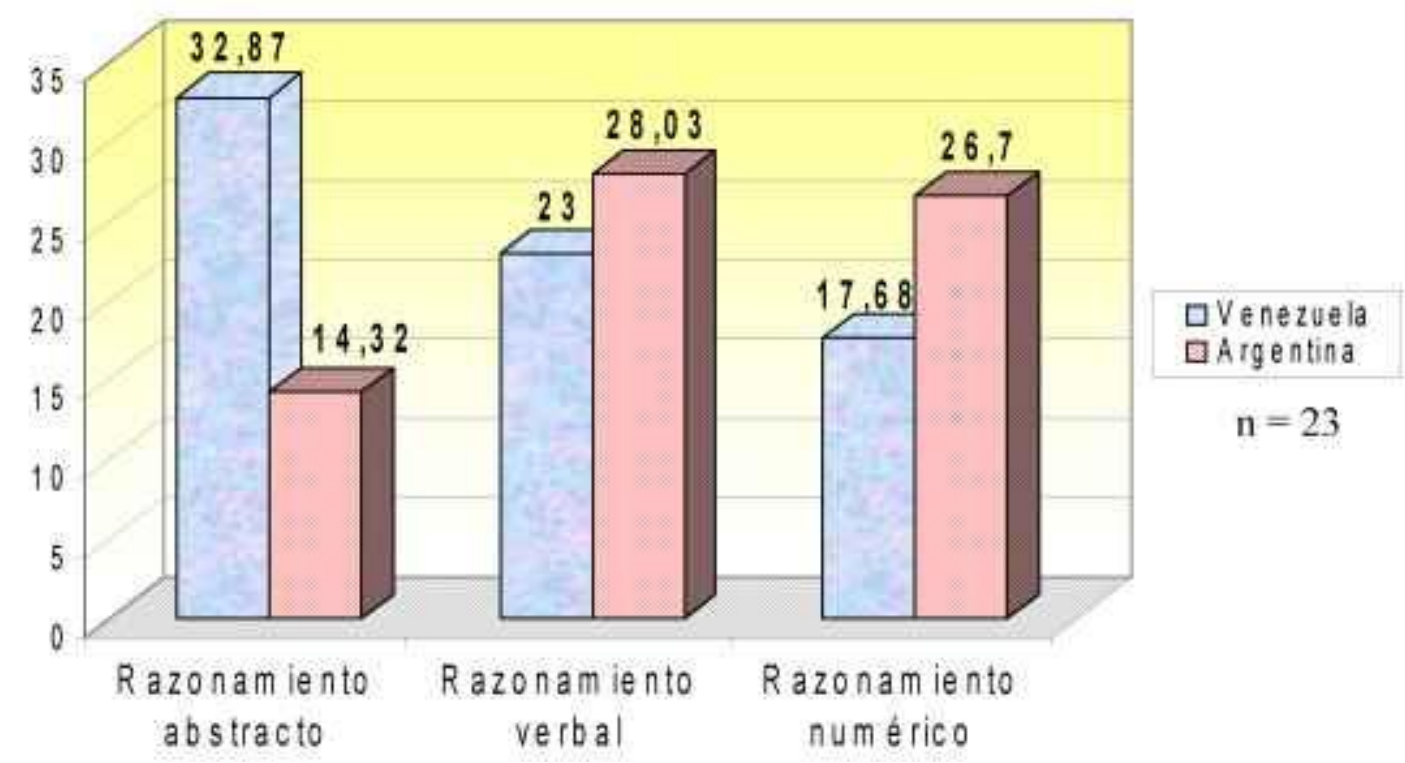

Fuente: instrumento aplicado

Con respecto a los factores de riesgo, evaluados a través del Drug Screening Use Inventory (DUSI), se obtuvo un índice de severidad total que evidencia diferencias considerables en el riesgo del consumo de alcohol en los y las estudiantes de ambos países. En este contexto, se obtuvo que $8,7 \%$ de los y las estudiantes venezolanos presentaron un índice de severidad por encima del 30\%, mientras que $59,9 \%$ de los y las estudiantes argentinos presentaron índices de severidad similares. En relación a los índices de severidad por área, se obtuvo en el factor correspondiente al área Conducta, que $26,1 \%$ de los y las estudiantes venezolanos presentan riesgo del consumo de drogas y $39 \%$ de los y las estudiantes argentinos presenta este tipo de riesgo al obtener índices de severidad superiores al $30 \%$.

Con respecto al riesgo asociado al Estado Emocional, 30,4\% de los y las estudiantes venezolanos presenta riesgo de consumo de alcohol y otras drogas, mientras $59,9 \%$ de estudiantes argentinos presenta riesgo de consumo de alcohol relacionado a esta área. El índice de severidad por área correspondiente al factor Competencia Social evidenció que $21,7 \%$ de los y las estudiantes venezolanos presenta riesgo de consumo de alcohol, mientras que $41,8 \%$ de los y las estudiantes argentinos presentaron riesgo en esta misma área al obtener índices de severidad por encima del $30 \%$. Se encontró que $8,7 \%$ de los y las estudiantes venezolanos encuestados tiene riesgo de consumo de alcohol y otras drogas influenciado por Factores Familiares, siendo una diferencia considerable con los y las estudiantes argentinos, de los cuales $41,8 \%$ presentaron riesgo de consumo de alcohol asociado a la familia.

En relación al riesgo del consumo de alcohol y otras drogas vinculado a factores Académicos se encontró que ningún estudiante venezolano presentó riesgo en esta área, y $5,5 \%$ de los estudiantes argentinos presentan este riesgo, al obtener índices de severidad superiores al $30 \%$. El índice de severidad por área correspondiente al factor influencia del Grupo de Pares evidenció que $13 \%$ de los y las estudiantes venezolanos presentó riesgo relacionado a este factor, al obtener $30 \%$ o más de respuestas afirmativas en el área, mientras que ninguno de los estudiantes argentinos presentó riesgo asociado a este factor al obtener índices inferiores a $30 \%$. Por otra parte, se obtuvo que $18,1 \%$ de los y las 
estudiantes argentinos presentaron riesgo relacionado a factores socioculturales, mientras esta área en la muestra de estudiantes venezolanos que participaron en el estudio fue un factor irrelevante al no manifestarse riesgo.

Tabla $\mathrm{N}^{\circ} 1$

Valores críticos del índice de severidad por área asociados al uso de drogas lícitas e ilícitas en estudiantes de enfermería venezolanos y argentinos

\begin{tabular}{|c|c|c|c|c|}
\hline \multirow{2}{*}{ Área } & \multicolumn{2}{|l|}{ Venezuela } & \multicolumn{2}{|l|}{ Argentina } \\
\hline & Frecuencia & $\begin{array}{l}\text { Valor } \\
\text { Critico }\end{array}$ & Frecuencia & $\begin{array}{l}\text { Valor } \\
\text { Critico }\end{array}$ \\
\hline Conducta & 17 & 73,9 & 9 & 39 \\
\hline Estado Emocional & 7 & 30,4 & 14 & 59,9 \\
\hline Competencia Social & 5 & 21,7 & 10 & 41,8 \\
\hline Familia & 2 & 8,7 & 10 & 41,8 \\
\hline Académico & 0 & 0 & 1 & 5,5 \\
\hline Grupo de pares & 3 & 13 & 0 & 0 \\
\hline Socio-culturales & 0 & 0 & 4 & 18,1 \\
\hline Recreación & 11 & 47,8 & 12 & 51,1 \\
\hline Uso del tiempo libre & 11 & 47,8 & 16 & 69,8 \\
\hline $\begin{array}{l}\text { Consumo problemático de } \\
\text { drogas }\end{array}$ & 0 & 0 & 1 & 2,2 \\
\hline $\begin{array}{l}\text { Indice de Severidad } \\
\text { Global }\end{array}$ & 2 & 8,7 & 14 & 59,9 \\
\hline
\end{tabular}

Fuente: instrumento aplicado

Respecto al factor Uso del Tiempo Libre, se obtuvo que $47,8 \%$ de los y las estudiantes venezolanos presentaron índices de severidad superiores a $30 \%$, mientras $69,8 \%$ de estudiantes argentinos presentaron niveles similares de riesgo de consumo de alcohol y otras drogas (Tabla $\mathrm{N}^{\circ} 1$ ).

En cuanto al área Consumo problemático de drogas, se obtuvo que ninguno de los y las estudiantes venezolanos presentaron índices de severidad superiores a $30 \%$, mientras $2,2 \%$ de los y las estudiantes argentinos presentaron riesgo de consumo de alcohol y otras drogas relacionados a este factor.

Con respecto a la relación entre pronóstico académico y factores de riesgo del consumo de drogas, se calcularon los coeficientes de correlación relacionando en primera instancia el pronóstico académico como una variable unitaria con el valor critico del índice de severidad global del DUSI, no encontrándose asociaciones estadísticamente significativas (tabla $\mathrm{N}^{\circ} 2$ ); sin embargo, llama la atención que cuando se procedió a calcular las correlaciones entre ambas pronóstico académico y factores de riesgo del consumo de drogas con enfoque multivariable, tomando cada factor de los que conforman las pruebas, es decir, las puntuaciones obtenidas en cada una de las subpruebas con los valores críticos de los índices de severidad por área en cada uno de los dominios, se encontró que sólo el factor de riesgo relacionado al área de conducta tuvo una correlación negativa estadísticamente significativa al $0,05(-0,174)$ con el razonamiento abstracto, $r^{2}=3,02 \%$, lo 
cual indica que los estudiantes que presentan mayor puntuación en razonamiento abstracto pudieran tener menor probabilidad de presentar factores de riesgo conductuales, tales como conducta impulsiva.

Tabla $\mathrm{N}^{\circ} 2$

Correlación entre pronóstico académico y riesgo de consumo de drogas por área

\begin{tabular}{|l|l|l|}
\hline Área & r de Pearson & $\mathbf{p}$ \\
\hline Conducta & 0,00 & 0,996 \\
\hline Estado Emocional & 0,032 & 0,712 \\
\hline Competencia Social & $-0,005$ & 0,954 \\
\hline Familia & 0,071 & 0,408 \\
\hline Académico & $-0,005$ & 0,951 \\
\hline Grupo de pares & $-0,020$ & 0,820 \\
\hline Socio-culturales & $-0,150$ & 0,077 \\
\hline Recreación & $-0,70$ & 0,414 \\
\hline Uso del tiempo libre & $-0,102$ & 0,236 \\
\hline $\begin{array}{l}\text { Consumo problemático de } \\
\text { drogas }\end{array}$ & $-0,54$ & 0,533 \\
\hline İndice de Severidad Total & 0,017 & 0,847 \\
\hline
\end{tabular}

Fuente: instrumento aplicado

\section{DISCUSIÓN}

Al comparar los resultados del estudio, se encuentra que los estudiantes venezolanos en promedio obtuvieron mejor puntuación en la subprueba de razonamiento abstracto y los estudiantes argentinos en las subpruebas de Razonamiento Numérico y Razonamiento Verbal, no apreciándose diferencias que fueran estadísticamente significativas.

Con respecto a los factores de riesgo, para los estudiantes venezolanos las áreas de riesgo son las correspondientes al factor conducta, asociado a impulsividad y falta de autocontrol; recreación, factor que implica el uso de drogas como una forma de recreación, y el uso de tiempo libre, que implica el inadecuado uso de tiempo en actividades de riesgo.

Para los estudiantes argentinos, los factores de riesgo más determinantes fueron el uso del tiempo libre, estado emocional y recreación; adicionalmente, los estudiantes argentinos reportaron mayor riesgo de consumo de alcohol y otras drogas que los venezolanos, apreciándose como uno de los factores más determinantes para el desarrollo de conductas adictivas.

Se observa que para ambos grupos de estudiantes la recreación y el uso del tiempo libre parecen ser los factores más relevantes para el inicio del consumo de sustancias, estos resultados coinciden con los hallazgos de estudios anteriores en países de Latinoamérica, como los encontrados en estudiantes universitarios argentinos, quienes atribuyeron como principal motivación para el consumo de bebidas alcohólicas la diversión y pasar el tiempo en las fiestas (1); así también, estudiantes colombianos manifestaron que el uso de drogas legales, como el alcohol y el cigarrillo forman parte del proceso de socialización dentro de las universidades (15). Los factores individuales, asociados al área de personalidad y desarrollo emocional también fueron relevantes como factores de riesgo tanto para los 
estudiantes de enfermería venezolanos como los argentinos, sin embargo, para los venezolanos el factor conducta es el principal, y para los argentinos el factor emocional fue el más importante, siendo que el primero está asociado a problemas con la impulsividad y el autocontrol, mientras que el segundo factor está relacionado con el desarrollo de trastornos afectivos y de aislamiento social. Cabe destacar que ambos factores de riesgo se han encontrado presentes en investigaciones realizadas con adolescentes que manifiestan consumir drogas, quienes presentan perfiles psicológicos caracterizados por un bajo control impulsivo, la tendencia a conductas auto y heteroagresivas (16), comportamientos de riesgo significativo en lo referente al manejo de la propia agresividad (17) siendo la morbilidad mental más común en estos pacientes jóvenes un grupo de patologías sociales constituido por trastornos de conducta, personalidad sociopática, adolescencia disfuncional y trastorno de personalidad limítrofe (17), coincidiendo estas investigaciones en la dificultad en el control de los impulsos.

Con respecto a la relación entre pronóstico académico y los factores de riesgo estudiados, se encontró que la capacidad para tener un pensamiento abstracto es un factor protector del consumo de drogas, esto quiere decir que en la medida en que los jóvenes sean capaces de comprender y analizar ideas más complejas, serán capaces de cuidar su salud y evitar comportamientos de riesgo, como el consumo de drogas. Este hallazgo concuerda con un estudio realizado en adolescentes colombianos en los que encontró que los participantes presentaban una insuficiente introspección o insigth, lo que dificultaba su recuperación en el tratamiento de rehabilitación por consumo de drogas, pues les dificultaba evidenciar las consecuencias negativas del consumo la droga (17). Así mismo, este resultado apoya la utilización de algunas estrategias para la prevención del consumo de drogas, específicamente en el enfoque de habilidades para la vida, en el que se incluyen las habilidades cognitivas como destrezas importantes para mantenerse alejados del consumo de drogas, siendo la solución de problemas una de las mayores habilidades que se identifica como un curso de acción que cierra la brecha entre la situación actual y una situación futura deseable. Este proceso requiere que quien toma la decisión sea capaz de identificar diferentes cursos de acción o solución a un problema, y determinar cuál es la mejor alternativa de solución, y para ellos es necesario tener desarrolladas las capacidades para el pensamiento abstracto (18).

Otro aspecto crucial de la cognición está relacionado con la autoevaluación o la capacidad de reflexionar sobre el valor de las propias acciones y las cualidades de uno mismo y con los demás, y también con la expectativa o el grado al que uno espera que sus esfuerzos den forma a la vida y determinen los resultados. Las personas que creen que son causalmente importantes en sus propias vidas tienen una tendencia a "participar en conductas más proactivas, más constructivas y saludables, las cuales se relacionan con resultados positivos." Se han establecido elementos correlativos entre este tipo de pensamiento y conductas tales como dejar de fumar, el uso de anticonceptivos entre las mujeres y los logros académicos (19)

Es así como se concluye en la necesidad de crear programas preventivos, basados en el enfoque de habilidades psicosociales, que desarrollen en los jóvenes la capacidad de pensar y tomar decisiones como una habilidad para la vida que les permitiría resistir las presiones de grupos características de esta edad. 


\section{REFERENCIAS BIBLIOGRÁFICAS}

(1) Porciel A. Percepciones y conductas de los jóvenes acerca del uso indebido de sustancias psicoactivas. Comunicaciones Científicas y Tecnológicas Universidad Nacional del Nordeste. [revista en Internet] [acceso 20 de Marzo 2009] Argentina. 2000 (1-4) disponible en http://www.unne.edu.ar/Web/cyt/cyt/2000/1 sociales/s pdf/s 031.pdf

(2) Cordini, Cohen, Santa Cruz, V. Liliana, V. Consumo de tabaco en estudiantes de medicina Revista de postgrado de la VI cátedra de medicina [revista en Internet] [acceso 22 de Marzo 2009] 169 (1-7) 2007 disponible en: http://med.unne.edu.ar/revista/revista169/2 169.pdf

(3) Zárate, M; Zavaleta, A; Danjoy, D; Chanamé, E. Prácticas de consumo de tabaco y otras drogas en estudiantes de ciencias de la salud de una universidad privada de Lima, Perú. ED Revista Investigación y Educación en Enfermería. Medellín, 24 (72-81). 2006.

(4) Martínez, G y Villar L, Margarita A. Estudio descriptivo del uso de drogas en adolescentes de educación media superior de la ciudad de Monterrey, Nuevo León, México. ED. Revista Latinoamericana Efermagem. [Revista en internet]. 12 Mar./Apr. 2004]. [acceso 15 de Enero 2009] disponible en http://www.scielo.br/scielo.php?pid=S0104-11692004000700014\&script=sci arttext

(5) González, Odris. Factores de riesgo en el consumo de drogas en los estudiantes de la escuela de arquitectura, universidad del Zulia, Maracaibo, Venezuela. [revista en internet]. 2000. Revista Multiciencia. 5 2005, [acceso 16 de Enero 2009] disponible en http://www.serbi.luz.edu.ve/scielo.php?script=sci arttext\&pid=S1317-

$22552005006000006 \&$ lng $=e s \& n r m=i s o$

(6) García, L., Fumero, A. (1998). Personalidad y rendimiento académico en estudiantes universitarios: Un estudio predictivo en tres cursos académicos. Análisis y modificación de conducta, 24 (93), 65-77

(7) Oficina de las Naciones Unidas contra las Drogas y el Delito (ONUDD) - OEA /CICAD. Jóvenes y drogas en países sudamericanos: Un desafío para las políticas públicas. ED. [sede Web] [acceso 16 de Enero 2009] disponible en http://www.cicad.oas.org/oid/NEW/Statistics/siduc/InfoFinal Estudio Comparativo.pdf

(8) Vélez van Meerbeke Alberto, Roa González Claudia Natalí. Factores asociados al rendimiento académico en estudiantes de medicina. Educ. méd. [Revista en Internet]. 2005 Jun [acceso 26 de Enero 2009]; 8(2): 24-32. Disponible en: http://scielo.isciii.es/scielo.php?script $=$ sci $\quad$ arttext\&pid $=\$ 157518132005000200005 \& \operatorname{lng}=e s \&$ nrm=iso.

(9) Hernandez De Rincón, A.l. El rendimiento académico de las matemáticas en alumnos universitarios. [Revista en internet]. abr. 2005, .12 (1) .9-30. [acceso 23 Enero 2009], Disponible en www.serbi.luz.edu.ve/scielo.php?pid=S1315$40792005004000002 \&$ script $=$ sci abstract

(10) Hernández, R; Fernández, C. y Baptista, P. Metodología de la investigación. 4ta edición. Mc Graw Hill México.2006

(11) Dawson-Saunders, B. y Trapp, R. Bioestadística médica. 2da ed. México DF: Manual moderno; 1997.

(12) Bennett, G. Bennet, N., y Clenderend, D.y otros APT test de pronóstico académico ediciones TEA; 1985.

(13) Bennett, G.k; Bennett M.G; Clendenen D.M; Doppelt J.E; Ricks, JH, Seashore, HG, Wesman, A:G. APT test de pronóstico académico manual (cuarta edición) Publicaciones de Psicología Aplicada TEA Madrid, 1996.

(14) Tarter R, Hegedus AM. The drug use screening inventory. Alcohol and research world 1991; 15: 65-75 
(15) Salazar, I.C, y Arrivillega, M. El consumo de alcohol, tabaco y otras drogas como parte del estilo de vida de los jóvenes universitarios. Revista colombiana de psicología 2004., 13, (74-89).

(16) Rees R, Valenzuela A. Características individuales y de la estructura familiar de un grupo de adolescentes abusadores de alcohol y/o marihuana. Rev. chil. neuro-psiquiatr. [Revista en Internet]. 2003 Jul [acceso 23 Marzo 2009] 41(3): 173-186. Disponible en: http://www.scielo.cl/scielo.php?script=sci arttext\&pid=S0717-92272003000300002\&lng =es

(17) Oliveira Magdalena Ribera, Luis Margarita Antonia Villar. Factores de riesgo para el consumo de alcohol en escolares de 10 a 18 años, de establecimientos educativos fiscales en la ciudad de La Paz - Bolivia (2003 - 2004). Rev. Latino-Am. Enfermagem [Revista en Internet]. 2005 [citado 05 de Junio 2009] ; 13 (spe): 880-887. Disponible en: http://www.revistasusp.sibi.usp.br/scielo.php?script=sci arttext\&pid=S01041169697620080 $00100008 \& \operatorname{lng}=$ pt. $2005000700016 \&$ Ing $=$ pt.

(18) Álvarez M, Cuervo M, Espinal M, Castaño J. Diagnósticos de enfermería, perfil social y clínico de adolescentes en tratamiento para la drogadicción en un centro de rehabilitación de Medellín 2006. SMAD, Rev. Eletrônica Saúde Mental Álcool Drog. (Ed. port.) [Revista en Internet]. 2008 Fev; 4 (1): [acceso 05 de Junio 2009 ]. Disponible en: http://www.revistasusp.sibi.usp.br/scielo.php?script $=$ sci $\quad$ arttext \&pid $=\$ 18066976200800010$ 0008\&lng $=$ pt.

(19) Mangrulkar, L, Vince, C, Posner, M Enfoque de habilidades para la vida para un desarrollo saludable de niños y adolescentes Organización Panamericana de la Salud División de Promoción y Protección de la Salud Programa de Salud Familiar y Población 2001. 